\title{
SYSTEMIC CREDIT RISK: WHAT IS THE MARKET TELLING US?
}

\author{
Vineer Bhansali* \\ Robert Gingrich* \\ Francis A. Longstaff**
}

\begin{abstract}
The ongoing subprime crisis raises many concerns about the possibility of much broader credit shocks in the economy. We use a simple linear version of the Longstaff and Rajan (2007) model to extract the information about macroeconomic credit risk embedded in the prices of tranches on the most-liquid credit indexes. Three types of credit risk appear to be priced by the market: idiosyncratic risks at the level of individual firms, sectorwide risk at the level of correlated firms within the same industry group, and economywide or systemic risk. We apply the model to the recent behavior of tranches in the U.S. and European credit derivatives markets and show that the current credit crisis has more than twice the systemic risk of the May 2005 auto-downgrade credit crisis.
\end{abstract}

Current version: March 2008.

* PIMCO, 840 Newport Center Drive, Newport Beach, CA 92660. ** UCLA Anderson School and NBER, 110 Westwood Plaza, Los Angeles, CA 90095-1481. 
The dramatic meltdown in the subprime market during the past year has raised many red flags among market participants about their potential exposure to broader systemic credit shocks. These heightened concerns have resulted in dramatic declines in market liquidity, restricted access to credit, flights to quality, sharply increased market volatility, and larger risk premia in many financial markets. As a result, the prices of the most credit-sensitive securities in the market may actually play the role of the "canary in the coal mine" in providing information about how market participants collectively assess the risk of systemic or macroeconomic credit shocks.

In this paper, we use the prices of indexed credit derivatives to extract market expectations about the nature and magnitude of the credit risks facing financial markets. Since their inception in 2002, indexed credit derivatives markets have exploded in size and participation. Broad indexes are now traded for U.S. (CDX) and European (ITRAXX) credit markets with usually very deep liquidity, and to a lesser degree for Japanese and U.K. credit markets. As of the end of 2007, the investment grade index was in its ninth generation, and its European counterpart was in its eight generation. What has been even more striking than the success of the indexes, however, is the launch and success of tranches on the indexes. Tranches can be best thought of as call spreads on the credit losses of a portfolio. Investors can use tranches to control their exposure to particular loss thresholds.

To extract the information from these credit derivatives, we first develop a simple linearized version of the collateralized debt obligation (CDO) pricing model of Longstaff and Rajan (2007). Longstaff and Rajan propose a three-jump model that is calibrated to the traded spreads of tranches and indexes directly. Their model allows for the possibility that credit spreads might be a composite of several different types of credit risk. Specifically, they find that the credit loss distribution embedded in index tranche prices includes a component for the risk of idiosyncratic or firm-specific defaults, a component for the risk of broader sectorwide or industrywide defaults, and a component for the risk of a massive economywide default scenario. We then fit the linearized version of the model to the market prices of the credit indexes and tranches. Their results are also consistent with the CDO modeling framework of Duffie and Gârleanu (2001).

The results have many important implications. Using current data for both investment-grade and high-yield indexes as well as for longer tenors, we likewise find that the market anticipates three different types of credit risks: idiosyncratic credit events, broader sectorwide credit events, and economywide credit events. What is particularly striking, however, is that the nature of systemic credit risk appears to have changed dramatically over time. In particular, systemic credit risk was only a small percentage of total credit risk during the auto-downgrade credit crisis of May 2005. In the recent subprime crisis, however, systemic credit risk has ballooned and now approximates the size of the idiosyncratic component of credit spreads. 
These results argue that the current credit crisis differs in fundamental ways from previous credit events. An important implication of this is that credit risk premia in financial markets may remain at high levels going forward, leading to a significantly higher cost of debt capital for many firms and sectors. Furthermore, this shifting trend in the nature of credit risk implies that traditional risk management strategies such as portfolio diversification may be less effective in controlling credit risk exposure.

Another key implication is that some of credit modeling tools that are widely used in practice may severely underestimate the actual risk exposure of credit portfolios. For example, during the May 2005 auto-downgrade crisis, many investors held positions in which the $0-3 \%$ equity tranche was hedged with the 3-7\% tranche based on the industrystandard Gaussian copula model. These trades performed completely differently than expected. The reason for this risk-management failure can ultimately be traced to a lack of liquidity and understanding by participants of the source of macro risks embedded in the tranches. Specifically, the behavior of index tranches to idiosyncratic and systemic risks varies significantly across their attachment and detachment points. Thus, while the Gaussian copula model can be applied to individual tranches, one correlation number is not sufficient to capture the risks of all tranches. At best, the implied base correlation of the copula model reflects the average correlation of the underlying equities, and hence, the correlation for the most junior or equity tranche (see Bhansali (2007)). Due to the lack of data on correlation during systemic defaults, it is difficult to extrapolate the tails to the higher senior part of the capital structure. Thus, using the copula model as a risk-management tool is fraught with dangers.

This paper is organized as follows. Section 1 presents the linearized CDO pricing model. Section 2 discusses the data and the methodology. Section 3 presents the results and discusses their implications for financial markets. Section 4 summarizes the results and makes concluding remarks.

\section{The Linearized Three-Jump Model}

Following Longstaff and Rajan (2007), let $L$ denote the proportion of portfolio losses realized on a credit portfolio. We write the proportion of portfolio losses as

$$
L=\gamma_{1} N_{1}+\gamma_{2} N_{2}+\gamma_{3} N_{3}
$$

where $L_{0}=0$, the $\gamma_{i}$ (where $i=1,2,3$ ) parameters denote jump sizes, and $N_{i}$ are independent Poisson counters that correspond to the number of jumps. Note that this allows for $L>1$, but in practice we never realize such large values of $L{ }^{1}$ In terms of

\footnotetext{
${ }^{1}$ The benefit of the computational speed from linearizing the model far outweighs this
} 
constant intensities $\lambda_{i}$ over a period $T$, we can write the probability of $j$ jumps for the $i$-th Poisson process $P_{i j}$ as as

$$
P_{i j}=\frac{e^{-\lambda_{i} T}\left(\lambda_{i} T\right)^{j}}{j !}
$$

The risk-neutral pricing equation for the index (e.g. the CDX) of maturity $T$ implies that the coupon $C$ for the index can be solved for by setting the value of the premium $\operatorname{leg}^{2}$

$$
C \int_{0}^{T} D(t)(1-E[L(t)]) d t
$$

equal to the value of the protection leg

$$
\int_{0}^{T} D(t) E[d L]
$$

Here $D(t)$ denotes the riskless discount factor for time $t$ (for pricing the CDX we use the swap curve) and the expected loss appears in the integral. ${ }^{3}$ Expanding the above expressions in terms of the loss function and Poisson intensities gives

$$
C \int_{0}^{T} D(t)\left(1-\gamma_{1} \lambda_{1} t-\gamma_{2} \lambda_{2} t-\gamma_{3} \lambda_{3} t\right) d t=\int_{0}^{T} D(t)\left(\gamma_{1} \lambda_{1}+\gamma_{2} \lambda_{2}+\gamma_{3} \lambda_{3}\right) d t
$$

After some straightforward algebra we can show that the index coupon value is given by

$$
C=\frac{\gamma_{1} \lambda_{1}+\gamma_{2} \lambda_{2}+\gamma_{3} \lambda_{3}}{1-\left(\gamma_{1} \lambda_{1}+\gamma_{2} \lambda_{2}+\gamma_{3} \lambda_{3}\right) A}
$$

technical difficulty with loss of accuracy. Furthermore, the probability of $L>1$ is vanishingly small for realistic calibrations.

${ }^{2}$ Although the formulas in the paper treat the cash flows from the index or the tranches as continuous for expositional simplicity, the numerical implementation of the model is based on discrete quarterly cash flows, consistent with the actual contractual provisions of these credit derivatives.

${ }^{3}$ As in Longstaff and Rajan (2007), we assume that interest rates are uncorrelated with loss realizations. 
where

$$
A=\frac{\int_{0}^{T} D(t) t d t}{\int_{0}^{T} D(t) d t} .
$$

In implementing the model, it will also be convenient to rearrange Equation (6) as

$$
\lambda_{1}=\frac{\frac{C}{1+A C}-\gamma_{2} \lambda_{2}-\gamma_{3} \lambda_{3}}{\gamma_{1}}
$$

Thus, given values of $\lambda_{2}$ and $\lambda_{3}$, Equation (8) may now be used to determine $\lambda_{1}$ as an explicit function of the market index spread $C$. This approach allows the model to fit the market index spread exactly. We use the index spreads and spreads on standard tranches to numerically obtain the values of $\lambda_{2}, \lambda_{3}$, and the jump parameters $\gamma_{1}, \gamma_{2}$, and $\gamma_{3}$.

\section{Data and Methodology}

To calibrate the model, we use the spreads for the CDX investment-grade and highyield indexes of various maturities and vintages. The data are obtained from PIMCO and J.P. Morgan. Since the CDX indexes roll every six months and come in various maturities (the most-liquid point being the five-year index followed by the ten-year and seven-year indexes), the investment-grade index derivatives markets are representative of the broad investment-grade U.S. credit market, while the same is true for the highyield index. The index derivatives suite also allows for market participants to implement "beta" and hedge views on the credit market directly, without taking the duration or liquidity risk that comes from using cash bond instruments. Similarly, we also use data for the ITRAXX index which tracks the credit risk of European investment-grade credit markets. ${ }^{4}$

In addition to the index data, we use data on the market spreads for standardized tranches on these indexes. The U.S. CDX investment-grade tranches are broken down

\footnotetext{
${ }^{4}$ Of course, the indexes are not completely sufficient to hedge or control for basis risk between cash market indexes (such as the Lehman corporate index), due to both composition differences and the cash-CDX basis, as well as the difference in liquidity risk embedded in the index and the individual names. For our study, however, these differences have negligible impact on our conclusions.
} 
in terms of losses than attach and detach at the $0 \%, 3 \%, 7 \%, 10 \%, 15 \%, 30 \%$, and $100 \%$ points. The index, by construction, is a $0 \%$ to $100 \%$ tranche. Note that the standard 0-3\% investment-grade equity tranche trades on a points-up-front basis with a fixed coupon of 500 basis points, while the other tranches trade based on a spread that changes with market conditions. The U.S. CDX high-yield tranches attach and detach at the $0 \%, 10 \%, 15 \%, 25 \%, 35 \%$, and $100 \%$ points. The $0-10 \%$ and $10-15 \%$ tranches trade on a points-up-front basis with zero fixed coupon, while the other tranches traded based on a market spread. Similarly, the European investment-grade ITRAXX index has tranches with points at $0 \%, 3 \%, 6 \%, 9 \%, 12 \%, 22 \%$, and $100 \%$. The $0-3 \%$ ITRAXX tranche likewise trades on a points-up-front basis with a 500 basis-point fixed coupon.

To see how we obtain the value of the parameters, first assume that the jump sizes $\gamma_{i}$ are given. For a tranche with attachment point $a$ and detachment point $b$, we can write the loss on the tranche $L_{a, b}(t)$ in terms of the loss function $L(t)$ on the underlying portfolio,

$$
L_{a, b}=\frac{\max (0, L-a)-\max (0, L-b)}{b-a} .
$$

This function illustrates that the losses on the tranche can be viewed as the payoffs for a call spread. Specifically, the losses on the tranche equal the payoff of a call on $L$ with strike $a$ minus the payoff of a call with strike $b$, where the spread is scaled by $1 /(b-a)$. As before, the tranche spread $C_{a, b}$ is determined by setting the value of the premium $\operatorname{leg}$

$$
C_{a, b} \int_{0}^{T} D(t)\left(1-E\left[L_{a, b}(t)\right]\right) d t
$$

equal to the value of the protection leg

$$
\int_{0}^{T} D(t) E\left[d L_{a, b}\right]
$$

To proceed further, we implement this pricing equation numerically. Specifically, we evaluate the expectations in Equations (10) and (11) by computing the tranche loss function $L_{a, b}(t)$ for values of $N_{i}$ ranging from zero to some suitably large value and then weighting by the corresponding Poisson probabilities $P_{i j}$ from Equation (2). This then allows us to solve for the model tranche spreads. ${ }^{5}$ To identify $\lambda_{1}$, we fit the model

\footnotetext{
${ }^{5}$ In fitting the model numerically, we allow for up to 50 jumps for the first Poisson process, up to 10 jumps for the second Poisson process, and up to 3 jumps for the third
} 
exactly to the market index spread $C$ using Equation (8). To identify $\lambda_{2}$ and $\lambda_{3}$, we minimize the root-mean-squared percentage pricing error between the model and the observed market tranche price for every day in the estimation period. Finally, we iterate over different values of the jump parameters $\gamma_{1}, \gamma_{2}$, and $\gamma_{3}$ until we achieve the global minimum root-mean-square percentage pricing error.

Once we have the $\lambda$ and $\gamma$ values, we can identify three different types of spreads which make up the full index spread in the risk-neutral setting:

$$
\begin{array}{r}
\text { Idiosyncratic } \equiv S_{1}=\frac{\gamma_{1} \lambda_{1}}{1-\left(\gamma_{1} \lambda_{1}+\gamma_{2} \lambda_{2}+\gamma_{3} \lambda_{3}\right) A}, \\
\text { Sectorwide } \equiv S_{2}=\frac{\gamma_{2} \lambda_{2}}{1-\left(\gamma_{1} \lambda_{1}+\gamma_{2} \lambda_{2}+\gamma_{3} \lambda_{3}\right) A}, \\
\text { Economywide } \equiv S_{3}=\frac{\gamma_{3} \lambda_{3}}{1-\left(\gamma_{1} \lambda_{1}+\gamma_{2} \lambda_{2}+\gamma_{3} \lambda_{3}\right) A}
\end{array}
$$

From Equation (6), we see that $C=S_{1}+S_{2}+S_{3}$. Thus, the sum of the three types of spreads equals the total spread of the index. Intuitively, our simple linear model is a way to decompose the index spread into its idiosyncratic, sectoral, and economywide components. Note that all computations are done in a risk-neutral setting, so we have no way of distinguishing how much of the spread might be due to risk premium terms.

Finally, to compute the sensitivity of a tranche with attachment $a$ and detachment $b$ to the spread $S_{i}$ (where $i=1,2,3$ ) as defined here, we numerically compute the change of a tranche price to a shift in the underlying spread.

\section{Results and Strategy Implications}

To implement the methodology described above in practice, we first fit the model to the index values and tranche spreads using current market data for the the CDX investmentgrade (IG), ITRAXX IG, and CDX high-yield (HY) indexes. Table 1 shows the results and parameter values from fitting the model.

Table 1 shows that there is considerable similarities in the results across indexes. The size of the first jump $\gamma_{1}$ is in the range of $0.9 \%$ to about $1.5 \%$ for all of the

\footnotetext{
Poisson process.
} 
investment-grade indexes, and roughly $2.3 \%$ for the high-yield indexes. Thus, a realization of the first Poisson process clearly can be given the interpretation of the idiosyncratic default of one or two of the firms in the index.

In contrast, the size of the second jump $\gamma_{2}$ ranges from about $6 \%$ to $10 \%$ across the different indexes. Since the firms in the CDX and ITRAXX indexes are roughly evenly distributed over, say, 10 to 12 broad industries or sectors, this second jump size is consistent with the realization of a credit event in which an entire industry or sector of the market goes into default.

Finally, the size of the third jump $\gamma_{3}$ ranges from about $50 \%$ to $75 \%$. Thus, for all indexes, a jump in the third Poisson process translates into credit event in which the majority of firms in the index default together, implying a catastrophic systemic credit event affecting all sectors of the economy. Clearly, the realization of such an event would be so severe that even the most senior index tranches would experience significant losses. These results parallel those reported in Longstaff and Rajan (2007) and extend the analysis using more-recent data for a broader set of indexes and maturities. ${ }^{6}$

In addition to the information about the nature and size of potential types of credit events, the model also allows us to infer the three components of the spread: $S_{1}, S_{2}$, and $S_{3}$. Figures 1 and 2 plot the values of these components for the CDX IG 8 five-year and ten-year indexes, respectively, for the March 2007 to December 2007 period. Figure 3 plots the ITRAXX 7 five-year index for the same period. Figure 4 plots the CDX HY 8 five-year index for the same period.

The four plots show clearly the onset of the subprime CDO crisis in mid-2007. Around the start of August 2007, all three components of the indexes begin to increase substantially. Interestingly, however, the individual components do not all increase by the same percentage. For the investment-grade indexes, the idiosyncratic component $S 1$ of the spread is roughly 50 to 75 percent higher during the second part of 2007 than its value during the first part of 2007. In contrast, the systemic component $S 3$ of the spread more than triples after August 2007. In fact, the systemic spread's value at the end of 2007 is nearly ten times its value in March 2007. The sectorwide component $S 2$ of the spread also increases during 2007, but by less than 10 basis points.

Figure 4 shows that a parallel situation holds for the CDX high-yield index. Overall, the values of the idiosyncratic and sectorwide components of the high-yield spread are only modestly higher during the latter part of 2007. The major difference occurs for the systemic component which rises dramatically from a level of about 25 basis points

\footnotetext{
${ }^{6}$ In our model, a realization of one of the Poisson processes might trigger the simultaneous default of multiple firms. A more general model, however, might allow for a cluster of defaults in a relatively narrow time interval, although not necessarily simultaneously. We are grateful to the referee for this suggestion.
} 
during the first part of 2007 to a range of about 100 to 150 basis points during the latter part of 2007.

These results provide valuable information about the market's credit concerns. By decomposing the credit indexes into three components, we see that much of the increase in credit spreads during 2007 is driven by concerns about the risk of systemic or macroeconomic credit problems. This is clearly a much different risk that, say, heightened concerns about the creditworthiness of individual firms, or even an entire industry.

To provide a longer-term perspective, Figure 5 plots the value of the three components of the spread based on fitting the model to the on-the-run CDX IG five-year index over the entire March 2005 to December 2007 period. In doing this, we are essentially treating the index as a continuous series. While the idiosyncratic risk component currently approximates its 2005 value, what is notable is that the systemic risk component is currently much larger in magnitude than in 2005. Systemic risk is also a larger proportion of the total risk of the index in late 2007 as compared to 2005.

This is logical, since in early 2005 the widening of the index was due to downgrades in the automotive sector. On the other hand, the 2007 spread widening can be traced to distress in the financial sector and a marketwide lack of liquidity, which arguably impacts the entire economy. Of course, the widening of the senior and supersenior tranches (such as the CDX IG 15-30\% tranche) means that more risk premium has come back into this part of the capital structure. There have been numerous headlines that suggest that the widening of the senior and supersenior tranches is due to the mark-to-market losses taken by specialized vehicle that had leverage exposure to these tranches. Note that this mark-to-market loss has indeed caused a weakened balance sheet for brokers and money center banks who effectively have financing exposures to many of these vehicles. Systemic risk is usually measured in terms of the widening of the Libor swap spread and the CDS spreads of banks and financials. It is thus no surprise that the same period in which the supersenior tranches have widened drastically in spread is accompanied by a sharp widening of swap spreads, financial sector spreads, increase in volatility, and general reduction in liquidity.

From a strategy angle, one would like to know what combination of tranches will most directly implement a view on one of the three macro spreads. To achieve this, Table 2 provides the exposures to the movements of the underlying spreads for the CDX IG five-year, seven-year, and ten-year spreads. To provide additional perspective, Table 2 also reports the sensitivities of the tranches to a one-basis-point move in the CDX index using the standard Gaussian copula model widely used on Wall Street.

As expected, the equity tranche has the largest exposure to the idiosyncratic risk factor, while the senior and supersenior tranches have higher exposure to the systemic factor. Thus, the tranches have very different sensitivities and their risk has a multi- 
dimensional nature. This has important risk management implications for these structured credit products. To illustrate, imagine that the CDX IG five-year index increases by a basis point. The standard copula model would imply that the price of the $0-3 \%$ equity tranche should change by 42.7 cents per $\$ 100$ notional. In our model, however, the change in the value of the equity tranche depends on the underlying source of the change in the CDX index. For example, if the change was entirely due to an increase in the idiosyncratic risk of the firms in the index, the valuation effect would be 90.5 cents instead. If the change was entirely due to an increase in the systemic component, the valuation effect would only be 2.2 cents. In the former case, the copula model would underestimate the valuation effect by more than 50 percent; in the latter case, the copula model would overestimate the valuation effect by a factor of more than 10 . The bottom line is that if index credit risk is really driven by three distinct factors, the use of a single-factor risk management measure is simply not adequate.

As an example of the use of these risk factors, assume that we thought that systemic risk would decline from its current elevated levels. So, if we wanted to go long (sell protection on) the economywide part of the index but remain unexposed to the idiosyncratic and sectorwide components while using five-year CDX IG tranches, we could go short credit (buy protection) on the 3-7\% and $7-10 \%$ tranches with notionals of $\$ 11.7$ million and $\$ 1.3$ million, respectively, and go long credit (sell protection) on the $0-3 \%, 15-30 \%$, and $30-100 \%$ tranches with notionals of $\$ 2.1$ million, $\$ 305$ million, and $\$ 2,282$ million, respectively. With this portfolio, a one-basis-point decline in the economywide spread would result in a profit of $\$ 1$ million, while moves in the other spreads would result in negligible profit and loss.

Credit curve risk premia may also be explained and exploited using this approach. In Figures 1 and 2, we show the allocation to the three spreads before and after the August 2007 subprime crisis for the five-year and ten-year CDX IG 8 indexes. Clearly, in both CDX IG indexes there is more systemic risk (systemic risk increased from 10 to $33 \%$ of the total five-year spread, while systemic risk increased from 16 to $30 \%$ of the total ten-year spread). In other words, even though the systemic risk embedded in the indexes increased, the increase was larger in the five-year index. Thus, to sell systemic liquidity premium, the five-year index and its tranches might currently be the preferred instruments.

\section{Conclusions}

In this paper, we show how the information in credit derivatives about the market's expectations of systemic credit risk can be extracted. We do this by implementing a simple linearized version of a three-jump model and calibrating it to market index and tranche spread levels. Thus, we are able to quantify the relative magnitudes of macro 
risks embedded in the liquid indexed credit derivatives. This also provides a unique way of expressing credit views with the increased menu of instruments. Furthermore, our approach provides a simple yet powerful framework for valuing tranches on bespoke portfolios relative to the prices of standard index tranches.

The results indicate that the current subprime credit crisis is fundamentally different from previous credit crises. Specifically, systemic credit risk has become a much larger fraction of total credit risk, while idiosyncratic and sectorwide credit risk levels have remained relatively constant throughout the current crisis. These results have many important implications for the financial markets. ${ }^{7}$

\footnotetext{
${ }^{7}$ We are grateful for helpful discussions with Arvind Rajan. This article contains the current opinions of the authors and not necessarily Pacific Investment Management Company LLC. These opinions are subject to change without notice. This article is distributed for educational purposes only and does not represent a recommendation of any particular security, strategy, or investment product. Information contained herein has been obtained from sources believed reliable, but not guaranteed. No part of this presentation may be reproduced in any form, or referred to in any other publication, without express written permission. Pacific Investment Management Company LLC, 840 Newport Center Drive, Newport Beach, CA 92660. Copyright 2008.
} 


\section{References}

Bhansali, Vineer 2008. "Correlation Risk-What the Market is Telling Us and Does it Make Sense?" in Credit Risk-Models, Derivatives, and Management, N. Wagner (ed.) Financial Mathematics Series, Vol 6, Chapman and Hall/CRC, Boca Raton, London, New York.

Duffie, Darrell, and Nicolai Garleanu 2001. "Risk and Valuation of Collateralized Debt Obligations." Financial Analysts Journal 57 (January/February): 41-59.

Longstaff, Francis A. and Arvind Rajan 2007. "An Empirical Analysis of the Pricing of Collateralized Debt Obligations." Journal of Finance, forthcoming. 
Table 1

Parameter Estimates and Root Mean Square Error Values from Model Fitting. This table reports the estimated jump size parameters $\gamma_{1}, \gamma_{2}$, and $\gamma_{3}$ (per $\$ 100$ notional) from fitting the model to the indicated index. Also reported are the percentage root mean square error (RMSE) $\gamma_{1}, \gamma_{2}$,
values.

\begin{tabular}{|c|c|c|c|c|c|c|}
\hline Index & Maturity & Horizon & $\gamma_{1}$ & $\gamma_{2}$ & $\gamma_{3}$ & RMSE \\
\hline CDX NA IG 8 & 5 years & $3-20-07$ to $12-31-07$ & 1.132 & 10.008 & 77.058 & 7.519 \\
\hline CDX NA IG 8 & 7 years & $3-20-07$ to $12-31-07$ & 1.048 & 9.265 & 62.802 & 5.083 \\
\hline CDX NA IG 8 & 10 years & $3-20-07$ to $12-31-07$ & 1.167 & 9.269 & 56.840 & 4.016 \\
\hline CDX NA IG 9 & 5 years & $9-21-07$ to $12-31-07$ & 0.914 & 9.303 & 62.496 & 4.368 \\
\hline ITRAXX Main 7 & 5 years & $3-20-07$ to $12-31-07$ & 1.511 & 9.143 & 61.265 & 6.674 \\
\hline ITRAXX Main 8 & 5 years & $9-20-07$ to $12-31-07$ & 1.254 & 9.206 & 55.865 & 4.267 \\
\hline CDX NA HY 8 & 5 years & $3-27-07$ to $12-31-07$ & 2.272 & 6.822 & 52.591 & 1.444 \\
\hline CDX NA HY 9 & 5 years & $9-27-07$ to $12-31-07$ & 2.314 & 6.343 & 50.599 & 1.048 \\
\hline
\end{tabular}


Table 2

Sensitivity of CDX IG Index Tranches to Changes in the Component Spreads of the CDX Index. This tables reports the DV01 or price change (per $\$ 100$ notional) in the indicated CDX index tranche CDOs with respect to a one-basis-point change in the index components, $S_{1}, S_{2}$, and $S_{3}$. Also reported is the DV01 based on the standard Gaussian copula model. The results are based on market prices for December 31, 2007.

\begin{tabular}{|c|c|c|c|c|c|c|c|c|}
\hline \multirow[b]{2}{*}{ Index } & \multirow[b]{2}{*}{ Maturity } & \multirow[b]{2}{*}{ DV01 } & \multicolumn{6}{|c|}{ CDX IG Index Tranche } \\
\hline & & & $0-3 \%$ & $3-7 \%$ & $7-10 \%$ & $10-15 \%$ & $15-30 \%$ & $30-100 \%$ \\
\hline \multirow[t]{4}{*}{ CDX IG 8} & 5 years & Copula & 0.427 & 0.277 & 0.099 & 0.055 & 0.027 & 0.010 \\
\hline & & $S_{1}$ & 0.905 & 0.394 & 0.017 & 0.024 & 0.001 & 0.001 \\
\hline & & $S_{2}$ & 0.178 & 0.387 & 0.405 & 0.177 & 0.008 & 0.001 \\
\hline & & $S_{3}$ & 0.022 & 0.049 & 0.052 & 0.052 & 0.053 & 0.037 \\
\hline \multirow[t]{4}{*}{ CDX IG 8} & 7 years & Copula & 0.401 & 0.406 & 0.185 & 0.111 & 0.051 & 0.015 \\
\hline & & $S_{1}$ & 0.944 & 0.744 & 0.078 & 0.065 & 0.004 & 0.002 \\
\hline & & $S_{2}$ & 0.180 & 0.542 & 0.583 & 0.276 & 0.022 & 0.002 \\
\hline & & $S_{3}$ & 0.023 & 0.077 & 0.085 & 0.087 & 0.088 & 0.046 \\
\hline \multirow[t]{4}{*}{ CDX IG 8} & 10 years & Copula & 0.315 & 0.537 & 0.302 & 0.176 & 0.082 & 0.023 \\
\hline & & $S_{1}$ & 0.724 & 1.260 & 0.429 & 0.136 & 0.021 & 0.005 \\
\hline & & $S_{2}$ & 0.143 & 0.621 & 0.770 & 0.536 & 0.068 & 0.005 \\
\hline & & $S_{3}$ & 0.016 & 0.094 & 0.120 & 0.127 & 0.132 & 0.060 \\
\hline
\end{tabular}




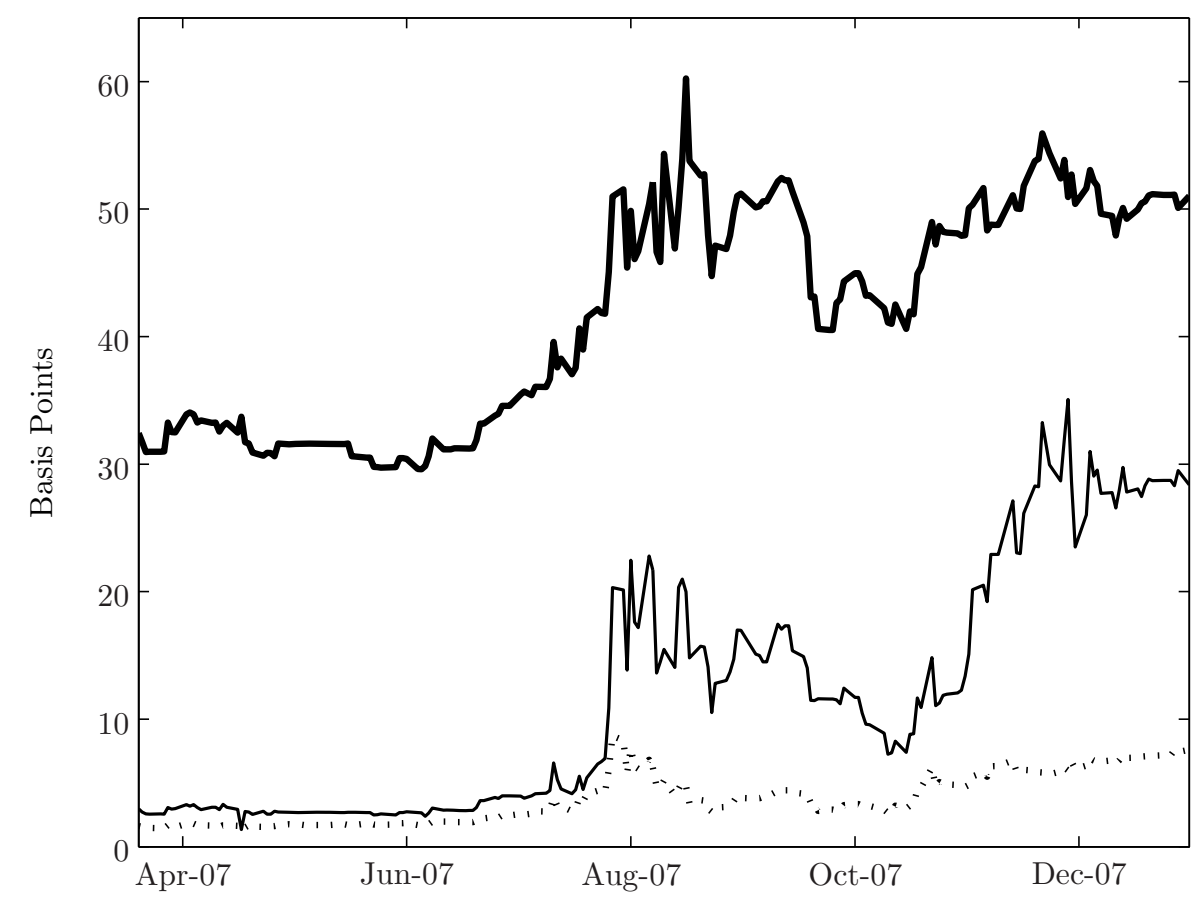

Figure 1. Components of the CDX IG 8 Five-Year Index.

The idiosyncratic component is represented by the darker solid line; the sectorwide component is represented by the dotted line; the economywide component is represented by the lighter solid line. 


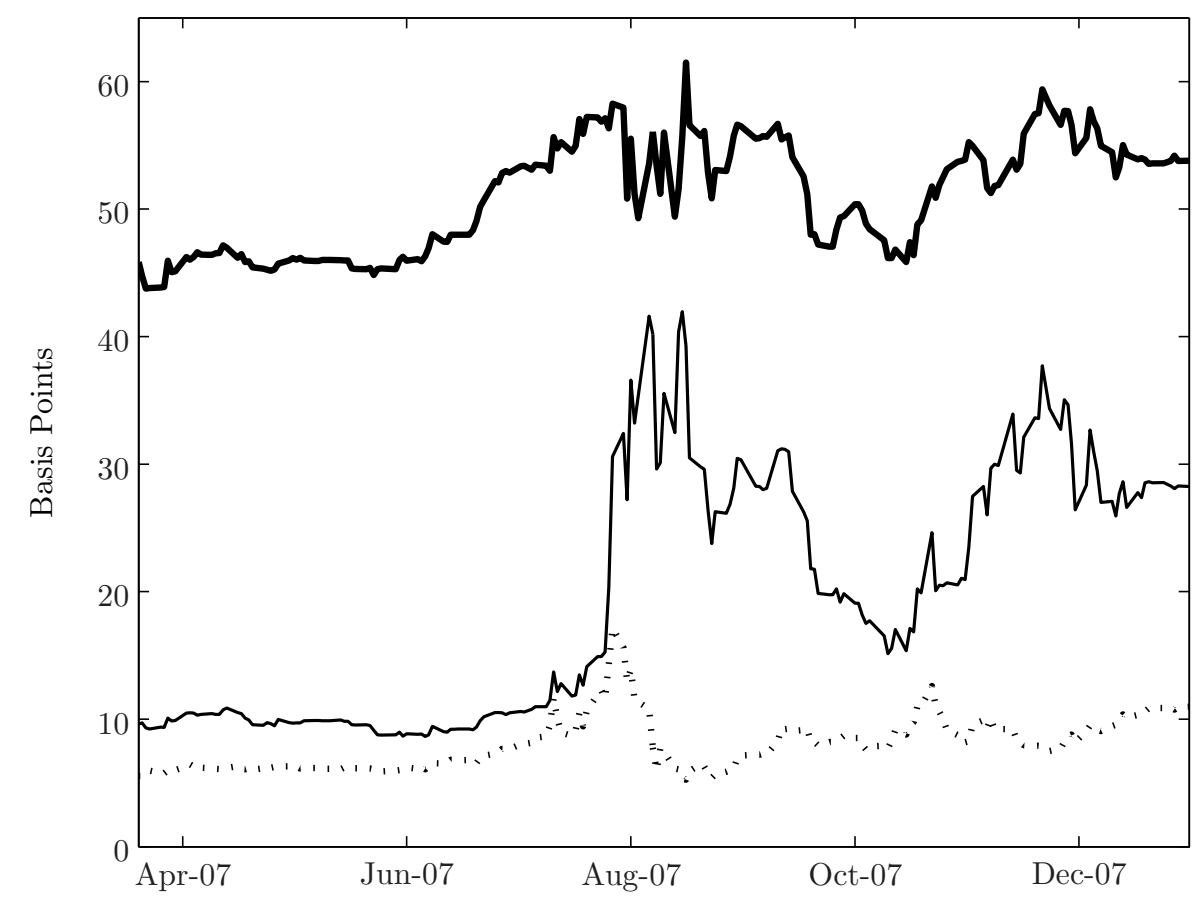

Figure 2. Components of the CDX IG 8 Ten-Year Index.

The idiosyncratic component is represented by the darker solid line; the sectorwide component is represented by the dotted line; the economywide component is represented by the lighter solid line. 


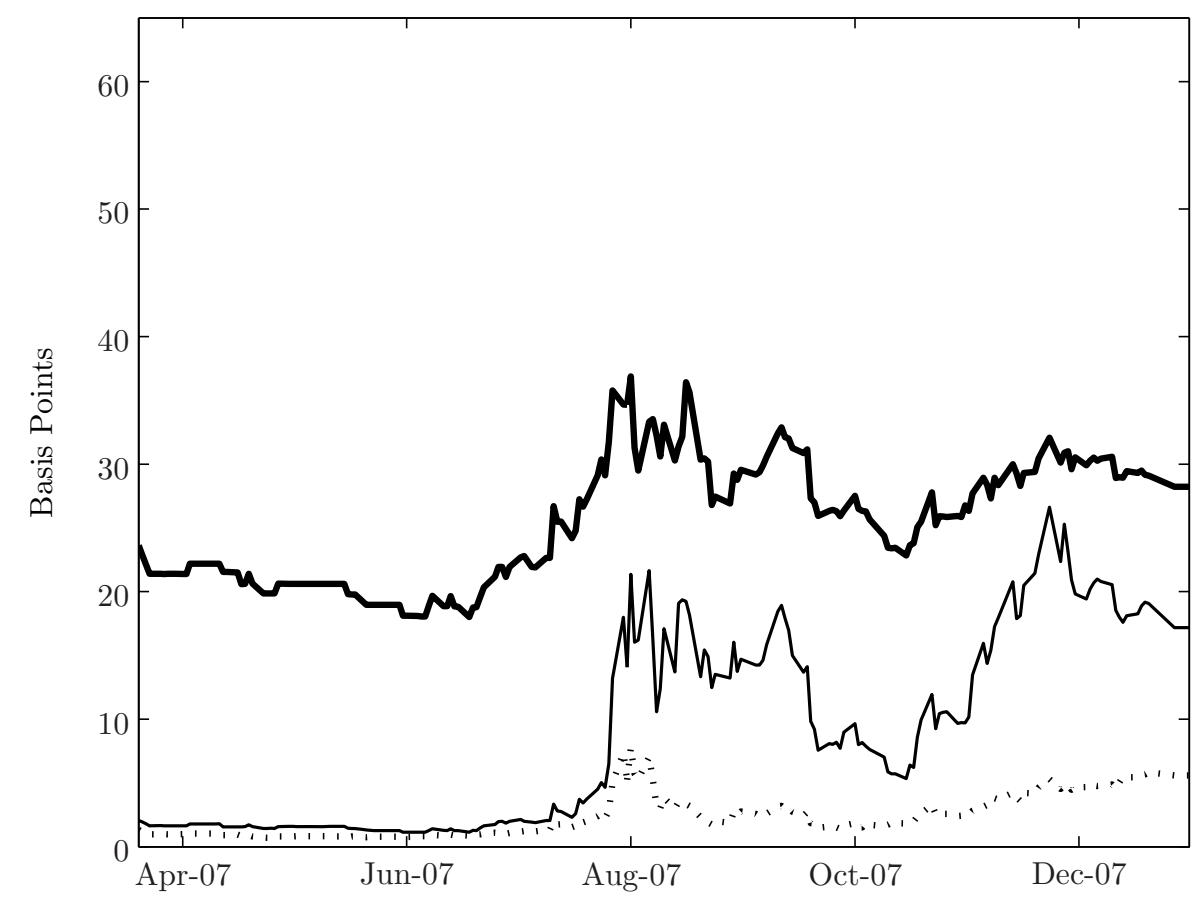

Figure 3. Components of the ITRAXX 7 Five-Year Index.

The idiosyncratic component is represented by the darker solid line; the sectorwide component is represented by the dotted line; the economywide component is represented by the lighter solid line. 


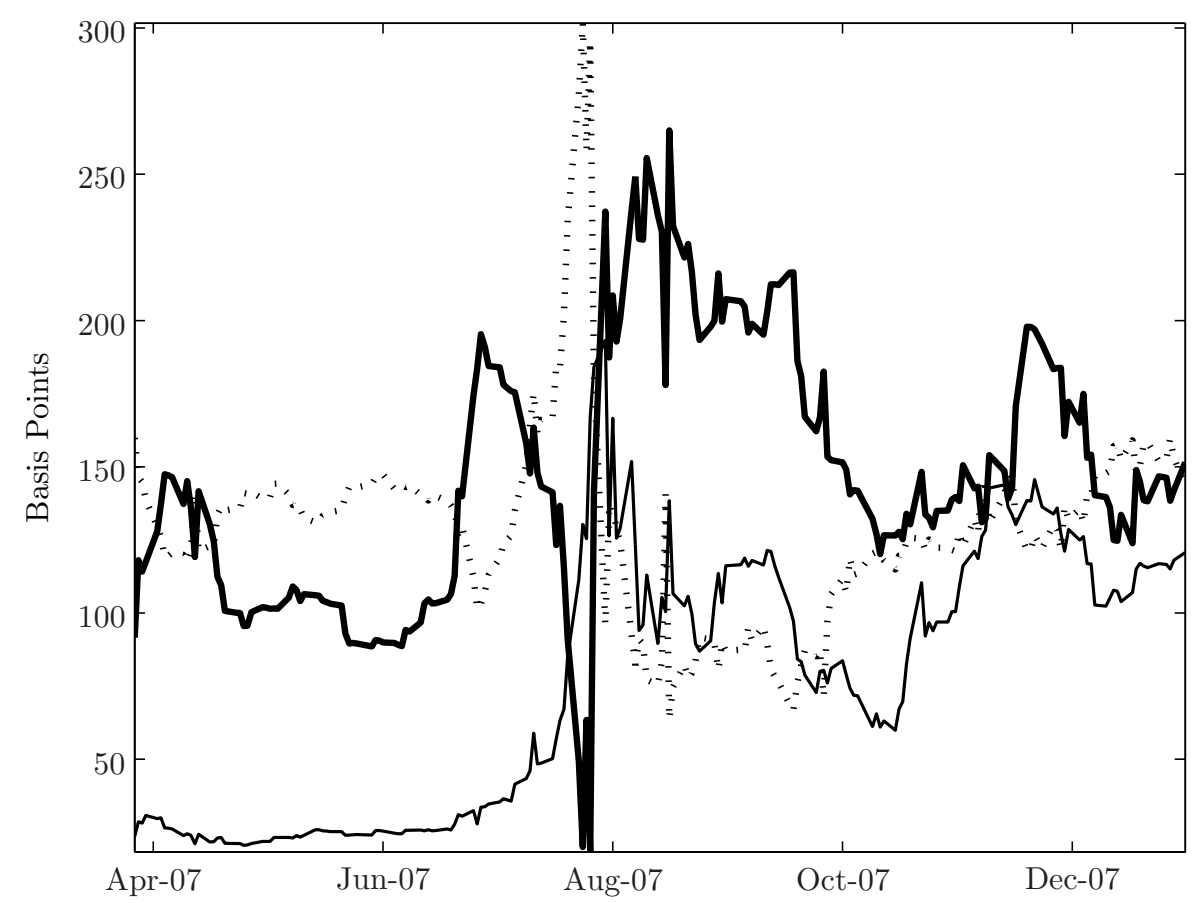

Figure 4. Components of the CDX HY 8 Five-Year Index.

The idiosyncratic component is represented by the darker solid line; the sectorwide component is represented by the dotted line; the economywide component is represented by the lighter solid line. 


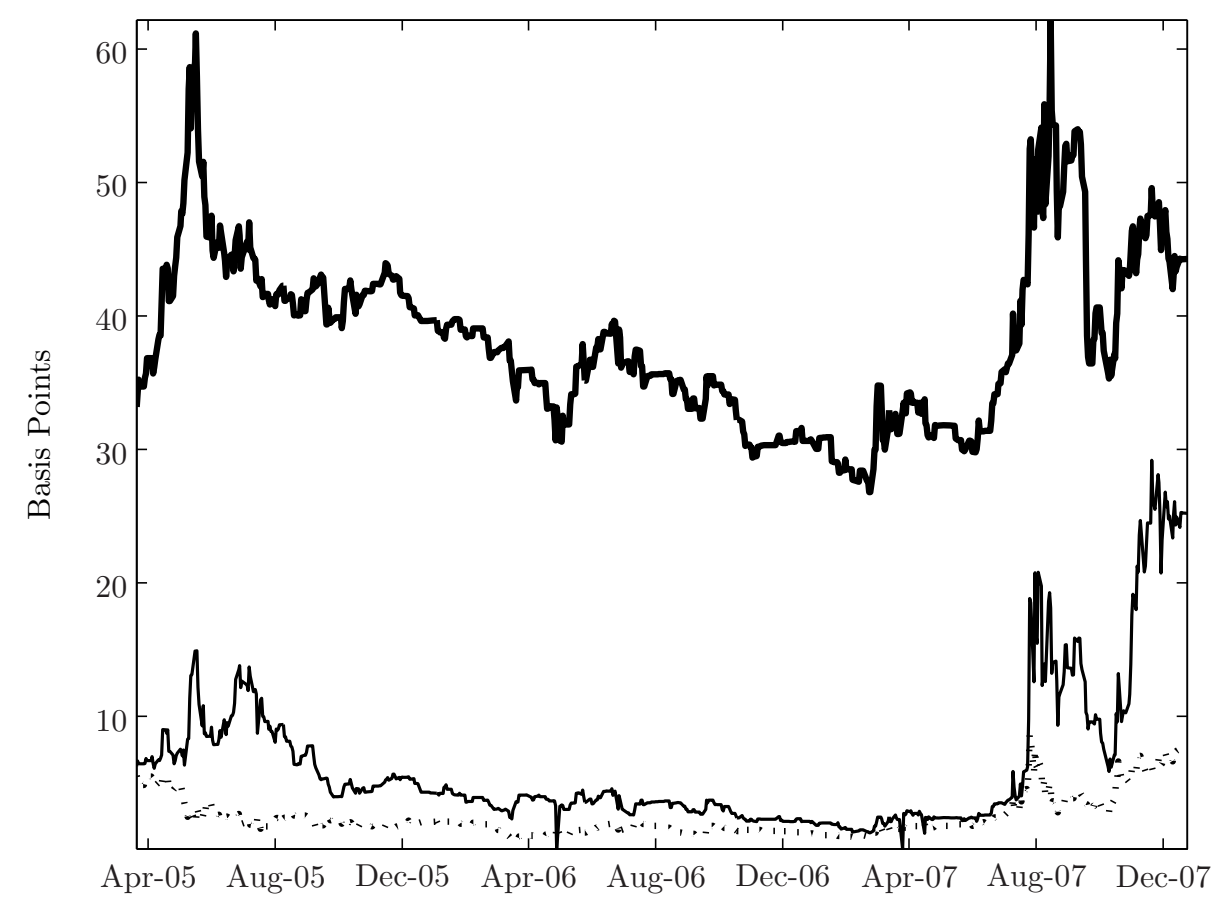

Figure 5. Components on the CDX IG Five-Year On-the-Run Index. This index is constructed by combining the on-the-run series from March 2005 to December 2007. The idiosyncratic component is represented by the darker solid line; the sectorwide component is represented by the dotted line; the economywide component is represented by the lighter solid line. 